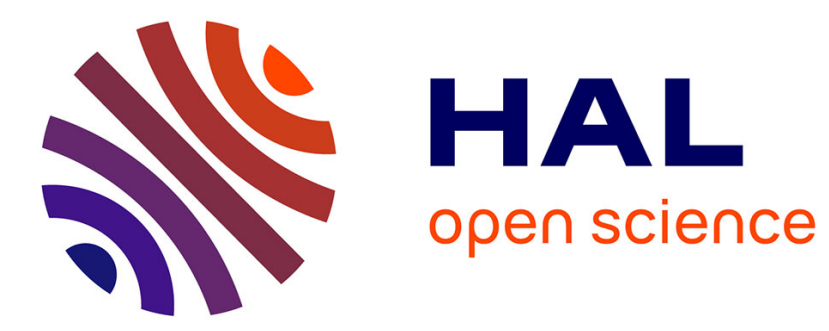

\title{
Hicks et l'économie de la dépression
}

Goulven Rubin

\section{- To cite this version:}

| Goulven Rubin. Hicks et l'économie de la dépression. 2011. halshs-00638187

\section{HAL Id: halshs-00638187 https://shs.hal.science/halshs-00638187}

Preprint submitted on 4 Nov 2011

HAL is a multi-disciplinary open access archive for the deposit and dissemination of scientific research documents, whether they are published or not. The documents may come from teaching and research institutions in France or abroad, or from public or private research centers.
L'archive ouverte pluridisciplinaire HAL, est destinée au dépôt et à la diffusion de documents scientifiques de niveau recherche, publiés ou non, émanant des établissements d'enseignement et de recherche français ou étrangers, des laboratoires publics ou privés. 


\section{Hicks et l'économie de la dépression}

Goulven Rubin ${ }^{*}$

\section{Résumé :}

Durant les années 1930, Hicks cherche à comprendre la crise à laquelle l'économie mondiale est confrontée. Mais à la différence de la plupart des économistes de l'époque, il tente de se représenter théoriquement la situation dans un cadre d'équilibre général walrassien. Le présent article étudie la façon dont Hicks a développé cette approche dans les derniers chapitres de Valeur et capital (1939). Hicks en vient à affirmer que les fluctuations du système capitaliste mettent son existence en danger et forme le projet d'une théorie des cycles qui accorderait une place centrale aux anticipations de prix et intégrerait un scénario d'effondrement du système. Les difficultés que ce projet rencontre dès 1939 sont analysées afin d'expliquer son abandon dans A Contribution to the Theory of the Trade Cycle (1950).

Mots clés : Hicks, Instabilité, Dépression. JEL : B22, E32, B21.

\section{Summary:}

During the 1930s, Hicks tried to understand the crisis to which capitalist systems were confronted. But unlike most economists of his time, he attempted to apply a Walrasian general equilibrium framework to the situation. The present paper studies how Hicks developed this approach in the last chapters of Value and Capital (1939). Hicks came to argue that the fluctuations of the capitalist system endangered its existence and imagined a theory of the trade cycle giving a major role to expectations and showing the possibility of an economic collapse. The paper analyses the problems raised by this research program in order to explain why Hicks abandoned it while he wrote A Contribution to the Theory of the Trade Cycle (1950).

Keywords: Hicks, instability, depression. JEL : B22, E32, B21.

\footnotetext{
* Goulven Rubin, LED et Université de Paris 8. Email : goulven.rubin@univ-paris8.fr. Adresse : 2, rue de la liberté 93526 Saint-Denis Cedex. Je voudrais remercier Carlo Benetti, Alain Béraud, Mauro Boianovsky, Michel De Vroey, Franco Donzelli, les participants du séminaire interne du LED ainsi que les deux rapporteurs anonymes de cette revue pour leurs commentaires sur des versions antérieures de ce texte. Je reste seul responsable des thèses développées.
} 


\section{Introduction}

"Few economists saw our current crisis coming, but this predictive failure was the least of the field's problems. More important was the profession's blindness to the very possibility of catastrophic failures in a market economy" (Krugman, P. New York Times, 6 septembre 2009).

"In periods of rapid change, elasticities of expectations are likely to be fairly large, beliefs of various individuals are likely to differ sharply, and the competitive mechanism may go astray in the sense that a temporary competitive monetary equilibrium may not even exist. The necessity of actually closing down financial markets in troubled times may be viewed as real life instances of the phenomenon." (Grandmont, 1991: 11)

Dans un point de vue daté de l'automne 2009, Paul Krugman nous alerte sur l'incapacité des économistes contemporains à penser « la possibilité même d'un échec catastrophique dans une économie de marché ». Penser la crise économique en termes d'échec catastrophique était en effet plus courant au sortir de la grande dépression, dans les années 1940. Mais cela n'allait pas sans difficulté, des difficultés qui peuvent nous aider à comprendre «l'aveuglement » des économistes d'aujourd'hui. La réflexion développée par Hicks au cours des années 1930 en offre une illustration.

Durant cette période, Hicks cherche à comprendre la crise à laquelle il est confronté. Mais à la différence de la plupart des économistes de l'époque, il tente de se représenter théoriquement la situation dans un cadre d'équilibre général walrassien. Cette approche le conduit à considérer la crise sous l'angle de l'instabilité de l'équilibre : un équilibre est supposé toujours exister mais l'économie peut ne pas être en mesure de l'atteindre. Cette idée apparaît sous sa plume en 1935 et constitue le fil rouge des derniers chapitres de Valeur et capital (1939). Il l'abandonnera cependant dans ses ouvrages ultérieurs.

Grandmont (1991) a souligné cette dimension de la contribution hicksienne à laquelle il a tenté d'offrir une traduction moderne dans ses propres travaux (Grandmont, 1974, 1983) ${ }^{1}$. Mais il n'a pas pris la peine d'analyser la façon dont Hicks se saisissait de cette idée. L'objet du présent article est donc l'analyse du programme de recherche esquissé par Hicks dans les derniers chapitres de Valeur et capital ainsi que les raisons de son abandon. La relation entre Valeur et capital et la Théorie générale de Keynes constitue un thème secondaire de la discussion. En 1939, Hicks tente de développer une alternative à «l'économie de la dépression » de Keynes, une économie de la dépression hicksienne.

Nous commencerons par montrer la façon dont la thèse défendue par Hicks sur la crise des années 1930 se radicalise entre 1933 et 1935 . Nous verrons comment il rompt avec l'idée que l'ajustement des salaires doit toujours conduire au rebond de l'économie (partie 1). Nous verrons ensuite comment Hicks en vient à affirmer que les fluctuations du système capitaliste mettent son existence en danger. Cette affirmation prend la forme d'un projet de théorie des cycles qui accorderait une place centrale aux anticipations de prix et intégrerait un scénario d'effondrement du système (partie 2). Nous étudierons ensuite les difficultés posées par l'intégration de l'instabilité de l'équilibre temporaire à la théorie des cycles et les limites de la théorie des anticipations à laquelle, pourtant, toute l'analyse de Hicks est suspendue (partie 3).

\footnotetext{
1 "In Value and Capital, Hicks was very much concerned by the potential instability of a temporary competitive equilibrium during the prices and interest rates adjustments that take place on a given "Monday", (Grandmont, 1991: 7).
} 


\section{Mr Hicks et les classiques}

Au cours des années 1930, Hicks a développé le projet d'une théorie de l'équilibre général qui ferait place au phénomène de la dépression. Deux textes témoignent de l'évolution de sa pensée. Le premier paraît dans une revue de langue allemande en 1933. Hicks appartient encore au camp des classiques mais il rompt avec l'explication hayekienne de la crise et pose les bases de sa réflexion. C'est dans le second texte, publié en septembre 1935, que surgit la problématique reprise dans les derniers chapitres de Valeur et capital. Pour penser la crise, la théorie doit envisager la possibilité que l'équilibre ne soit pas atteignable ${ }^{2}$.

\subsection{Un point de départ presque entièrement classique}

«Equilibrium and the Cycle»(1933) contient déjà la problématique qui conduit à la théorie de l'équilibre temporaire. Hicks entend améliorer le concept d'équilibre stationnaire légué par Pareto ${ }^{3}$. Il propose de le remplacer par un concept «d'équilibre dynamique », ce qu'on appellera par la suite le concept d'équilibre intertemporel. Le temps est découpé en périodes durant lesquelles les fondamentaux de l'économie changent «d'une façon qui a été prévue ». Sa caractéristique essentielle est que les anticipations de prix initiales se réalisent, elles sont «parfaites». Cette définition d'un «système économique qui fonctionne parfaitement » met en évidence la clé des dysfonctionnements observés dans la réalité, l'imperfection des anticipations :

"Because of ignorance of future changes of data (and still more of the consequences of changes of data - not only of future or present changes, but also of those that have already occurred in the past) such perfect equilibrium is never attainable. A real economy is always in disequilibrium » (1933: 32).

Ce qui manque à ce stade est une théorie capable de rendre compte du «déséquilibre » tel que le définit Hicks. Mais cela ne l'empêche pas d'aborder la question du cycle des affaires. Dans un contexte dépourvu d'incertitude, tous les agents désireraient prêter leur monnaie. La demande de monnaie serait donc nulle. Cette demande dérive de l'incertitude concernant les dates des paiements futurs. Sur cette base, le cycle est conçu comme l'effet de chocs modifiant le niveau d'incertitude et affectant la demande de monnaie. Lorsque l'incertitude s'accroît, les agents sont conduits à accroître la part de la monnaie dans leur portefeuille d'actifs et réduisent leurs achats de capital. Cela déclenche la récession. Cette approche implique une rupture par rapport à la position de Hayek. Le cycle n'est pas nécessairement la conséquence d'une politique monétaire inappropriée : "Even a system of pure laissez faire would be subject to monetary disturbances » (1933 : 35). Hicks reste cependant classique en affirmant que l'ajustement des salaires à la baisse assure le rebond de l'économie. Si la dépression persiste, les syndicats sont à blâmer :

"[W]hen depression has taken root, and expressed itself in a sequence of business losses, the natural way out is by a reduction in costs. I do not mean to imply that every Depression must end that way; a favourable change in data may make it unnecessary. But a reduction in costs is the only step that arises naturally out of the process and that is favourable to recovery. Under free competition, such a reduction will occur - in the end; but if the labour market is not freely competitive - wage-reductions being resisted by Trade Unions - the position is doubly difficult. The period of adaptation is lengthened while risk is increased by labour unrest » (1933: 37).

\footnotetext{
${ }^{2}$ Sur la question plus générale du rapport que Hicks entretient avec les concepts walrassiens d'équilibre au cours des années 1930, voir Béraud (2008) et Donzelli (2009).

${ }^{3}$ Hicks considère que l'application du concept d'équilibre par Pareto est supérieure à ce que l'on trouve chez Walras. Sur ce point, voir Donzelli (2009).
} 


\section{2 «Un équilibre désespérément instable »}

«A Suggestion for Simplifying the Theory of Money (1935) marque un tournant dans la façon dont Hicks envisage la dépression. Lui-même a présenté ce texte comme une « déclaration d'indépendance » à l'égard de «l'école du libre marché » (Hicks, 1982: 10). Désormais, il considère que l'ajustement des salaires n'assure plus le rebond de l'économie. Seules les anticipations des ménages modestes empêchent le système capitaliste de s'effondrer. Mais surtout, Hicks cherche à inscrire cette vision du capitalisme dans un cadre théorique. Il tente de répondre d'une manière à la fois originale et radicale à une question implicite : comment penser une récession qui dégénère dans le cadre de la théorie de l'équilibre général ?

L'enjeu principal de la «Suggestion » est bien connu. Il s'agit d'intégrer la théorie de la monnaie à la théorie de la valeur en lui appliquant les principes de la théorie des choix marginaliste ${ }^{4}$. Hicks prend appui sur le Treatise on Money de Keynes pour poser les bases de la théorie des choix de portefeuille qui sera développée dans les années 1950 par Harry Markowitz et James Tobin. Mais l'article contient aussi une charge contre la théorie quantitative de la monnaie, charge qui ouvre sur la question du cycle. Hicks soutient que les «théoriciens de la monnaie » supposent en général une relation proportionnelle entre la demande de monnaie et la «valeur nette des actifs» détenus par les agents. Dans ces conditions, le «système exhiberait une sorte de stabilité plutôt simple » (1935: 15). Bien que très sommaire, le raisonnement de Hicks suppose un cadre d'équilibre général et s'appuie sur une loi de Walras restreinte aux marchés d'actifs. Un excès d'offre de monnaie impliquerait un excès de demande sur les marchés des biens capitaux et des titres. La hausse du prix de ces biens génèrerait mécaniquement une hausse de la demande de monnaie qui rétablirait l'équilibre. Mais les «théoriciens de la monnaie» ont le défaut d'oublier les anticipations. Une hausse de la «richesse » des agents les conduit à demander davantage de monnaie. Cependant, elle entraîne aussi une révision à la hausse du «rendement anticipé des investissements » qui réduit la demande de monnaie. Hicks soutient que dans le cas où ces deux effets viendraient à se compenser, l'équilibre général de l'économie deviendrait « désespérément instable » :

"We are treading on thin ice; but the unpleasant possibilities which now begin to emerge are sufficiently plausible for their examination to be well worth while. What happens, to take a typical case, if the demand for money is independent of changes in wealth, so that neither an increase in wealth nor a diminution will affect the demand for money?

One can conceive of a sort of equilibrium in such a world but it would be a hopelessly unstable equilibrium » (1935: 16).

Et dans ces conditions, ajoute Hicks : «It will not surprise us to be told that wage-changes will avail nothing to stop either and inflation or a deflation » $(1935: 17)$.

Dans son texte de 1933, Hicks discute en détail le phénomène de la récession mais il insiste alors sur le caractère «naturel » du retour au plein emploi. Désormais, il rejette une approche qui «seems to make the economic system work much too smoothly to account for observed fact » (1935: 15). Il s'intéresse à un cas dans lequel l'accroissement de la demande de monnaie fait s'effondrer les prix «jusqu'à zéro ». Il écrit que l'ajustement des salaires peut ne pas stopper la déflation. En d'autres termes, l'enjeu est maintenant de comprendre, et cela dans un cadre d'équilibre général, pourquoi la récession peut dégénérer. La solution qu'il propose est originale. Une récession qui dégénère correspond en théorie à un équilibre général

\footnotetext{
${ }^{4}$ Pour une critique de l'approche de Hicks, sur ce point, et de la tradition issue de son article, voir Benetti (2001).
} 
instable. Implicitement, Hicks suppose que l'équilibre existe. L'instabilité correspond alors à l'impossibilité d'atteindre cet équilibre en un point du temps. Dans le texte de 1935, Hicks donne deux interprétations économiques à cette instabilité. Il évoque alternativement des «fluctuations désespérées », qui laissent à penser que le système fonctionne mal mais fonctionne, et un « effondrement du capitalisme » («break down ») plus radical.

Le scénario d'un équilibre instable capture bien certaines caractéristiques du capitalisme aux yeux de Hicks. Mais ce dernier s'empresse d'ajouter qu'il n'est pas suffisamment réaliste. Une théorie intermédiaire entre les hypothèses de la théorie quantitative et l'hypothèse d'une demande de monnaie constante est requise :

"These exercises in the economics of an utterly unstable world give us something too mad to fit even our modern Spatkapitalismus ; but the time which economists have spent on them will not have been wasted if they have served as a corrective to the too facile optimism engendered by the first assumption we tried. Obviously, what we want is something between the two - but not, I think, a mere splitting of the difference" (1935: 17).

Hicks se contente d'esquisser la théorie intermédiaire. L'hypothèse clé serait la répartition de la population en deux groupes aux comportements de demande de monnaie hétérogènes. Compte tenu des coûts de transaction, seuls les agents disposant d'un patrimoine suffisant investissent et sont sensibles aux variations anticipées des taux de rentabilité. Les ménages pauvres qui disposent d'une «proportion considérable du stock de monnaie de la communauté » sont «insensibles à l'évolution des anticipations» (1935: 17). En conséquence, la demande de monnaie des plus riches peut être décroissante par rapport à la « richesse » alors que celle des plus pauvres est généralement croissante. Le comportement des plus riches expliquerait les fluctuations cycliques, celui des plus pauvres expliquerait pourquoi ces fluctuations ne sont pas explosives. Hicks en déduit que le développement du capitalisme, en réduisant les coûts d'accès aux «actifs », accroît le nombre d'agents sensibles et devient par conséquent de plus en plus instable. Le système est «son propre ennemi $»^{5}$. Mais on note que dans la théorie « réaliste » qu'il envisage, l'instabilité se manifeste sous la forme de fluctuations cycliques. L'effondrement du système («break down ») n'est qu'un cas d'école.

\section{L'économie de la dépression dans Valeur et capital}

Dans Valeur et capital, Hicks reprend et radicalise la thèse esquissée en 1935. Dans les derniers chapitres de l'ouvrage, il présente l'instabilité de l'équilibre temporaire comme un danger auquel serait réellement confronté le système capitaliste de son époque. La possibilité d'un «échec catastrophique du système de marché », pour reprendre l'expression de Paul Krugman, se trouve au centre de son élaboration théorique. Peut-on parler à ce sujet d'une économie de la dépression? Hicks applique cette expression à la théorie de Keynes dans «Mr. Keynes and the Classics » ${ }^{6}$. Il ne l'emploie pas dans Valeur et capital. Mais le scénario qu'il met en avant est bien la version hicksienne de la théorie qu'il attribue à Keynes en 1937.

\footnotetext{
${ }^{5}$ «If it is the insensitive people who preserve the stability of capitalism, people who are insensitive (you will remember) largely because for them the costs of transferring assets are large relatively to the amount of assets they control, then the development of capitalism, by diminishing these costs, is likely to be a direct cause of increasing fluctuations. It reduces costs in two ways : by technical devices (of which banks are only one example), and by instilling a more 'capitalistic' spirit, which looks more closely to profit, and thus reduces subjective costs. In doing these things, capitalism is its own enemy, for it imperils that stability without which it breaks down" (1935b: 18).

${ }^{6}$ «So the General Theory of Employment is the Economics of Depression » (Hicks, 1937: 155).
} 
Comme Keynes, Hicks étudie les conditions qui conduisent à l'aggravation d'une récession. Cependant, l'approche qu'il propose se démarque à plusieurs niveaux. Notre objectif est de dégager cette alternative hicksienne contenue dans le texte de 1939. Car la proposition formulée par Hicks ne se laisse pas saisir aisément. Elle est encastrée dans une argumentation tortueuse et torturée.

\subsection{Les conditions de l’instabilité}

La possibilité théorique d'une instabilité de l'équilibre temporaire, évoquée en quelques lignes seulement dans la «Suggestion » de 1935, est le sujet de deux chapitres au moins de Valeur et capital. Hicks présente alors comme «la proposition la plus importante de l'économie dynamique » $(1939$ : 255) la thèse selon laquelle un système à prix et salaires flexibles caractérisé par une élasticité des anticipations supérieure ou égale à l'unité pourrait se révéler instable.

Hicks élabore le concept d'équilibre temporaire dans le prolongement d'un article intitulé «Wage and Interest: the Dynamic Problem»(1935) ${ }^{7}$. L'objet de la théorie de l'équilibre temporaire est d'étudier la dynamique d'une économie monétaire. Cette dernière est conçue comme un système d'équilibre général avec monnaie et titres. Hicks suppose que le temps est découpé en semaines. Le lundi de chaque semaine, un ensemble de prix d'équilibre est déterminé pour lequel des contrats d'achats et de ventes cohérents sont signés. Le reste de la semaine est consacré à l'exécution des contrats, c'est-à-dire à la production et à l'échange des marchandises sans que les prix soient révisés. Dans ce contexte, les consommateurs et les entreprises déterminent leurs offres et leurs demandes pour la période courante mais aussi pour l'ensemble des périodes futures. Ce «plan » est donc fonction des prix courants et des prix futurs. En l'absence de marchés à terme, du fait de l'existence d'une incertitude concernant l'avenir, les prix futurs doivent être anticipés. L'équilibre est alors temporaire dans la mesure où il n'y a aucun raison que les anticipations de prix se réalisent. Enfin, la dynamique de l'économie est conçue comme une séquence d'équilibres temporaires.

Hicks décompose l'analyse des conditions de stabilité de l'équilibre en deux étapes. Il suppose d'abord le taux d'intérêt constant et soutient que la stabilité dépend du rapport entre prix futurs et prix courants, rapport mesuré par «l'élasticité des anticipations de prix » (chapitre 20). Si les anticipations sont inélastiques, un excès de demande de biens qui génère une élévation du niveau des prix produit un effet de substitution intertemporelle stabilisant. Les prix des biens présents s'élèvent par rapport au prix des biens futurs et la demande de ces derniers augmente par rapport à la demande des premiers. Les choses se compliquent dans le cas où l'élasticité est égale à 1 , une hypothèse « si plausible qu'elle a été tenue pour évidente [taken for granted] par la majorité des économistes » (1939: 251). L'apparition d'un excès de demande pour une marchandise $\mathrm{X}$ conduit à une hausse de son prix. Si tous les prix (présents et futurs) augmentent dans les mêmes proportions, on retrouve le même système de prix relatifs. L'équilibre est préservé sur les autres marchés mais l'excès de demande persiste sur le marché du bien X. Les prix vont donc continuer à augmenter sans fin. Hicks conclut que le système est « imparfaitement stable » (instable mais pour un taux d'intérêt supposé constant). Cette instabilité est encore aggravée si l'élasticité des anticipations de prix est supérieure à 1 . Dans la seconde étape de l'analyse (chapitre 21), il soutient que l'ajustement des taux d'intérêts ne suffit pas à restaurer la stabilité (1939 : 258-63). L'argument, inspiré par Keynes,

\footnotetext{
${ }^{7}$ Dans l'article «Wages and Interest: The Dynamic Problem» (1935a) Hicks présentait un modèle d'équilibre temporaire à trois biens : pain, travail et titres. Dans ce cadre, il analysait les effets d'une variation des salaires à la baisse puis à la hausse. Les conclusions étaient parfaitement classiques. Une baisse des salaires devaient généralement provoquer une hausse de la demande de travail. Donzelli (2009) souligne que le concept d'équilibre temporaire était déjà présent chez Pareto et Walras et essaye d'expliquer pourquoi Hicks ne le voit pas et affirme qu'il le doit aux suédois Myrdal et Lindahl.
} 
est celui de la trappe à liquidité. En situation d'excès d'offre de biens, la chute des prix réduit la demande de monnaie et, par conséquent, le taux d'intérêt. Mais le taux d'intérêt ne peut pas baisser en dessous du niveau qui compense le risque et les coûts de transaction associés à la détention de titres. Or le taux d'intérêt qui permettrait le retour à l'équilibre peut très bien être inférieur. Hicks développe l'argument en distinguant taux courts et taux longs et en faisant intervenir la structure par terme des taux d'intérêts. Si le marché détermine un taux court, l'évolution du taux long, dont dépendent les décisions de consommation et d'investissement, est fonction de l'élasticité des taux courts anticipés. Or, d'après Hicks, il y a de bonnes raisons de penser que cette élasticité est faible. Dans ce cas, il est possible que seul un taux d'intérêt négatif inaccessible garantisse l'équilibre ${ }^{8}$. Hicks ajoute un dernier facteur d'instabilité sous la forme des effets de redistributions associés à la déflation, argument important de Fisher (1933) et du chapitre 19 de la Théorie générale (1939: 264).

L'analyse des chapitres 20 et 21 de Valeur et capital introduit donc la possibilité d'une instabilité de l'équilibre temporaire. A ce stade de l'ouvrage, Hicks ne distingue pas explicitement la question de l'existence de l'équilibre de celle de sa stabilité. Cette distinction est cependant implicite. Dans les chapitres 4 et 5 , Hicks sépare le problème de la «détermination» de l'équilibre général (le décompte des équations et des inconnues) et le problème du «fonctionnement » ou de la «stabilité » de l'équilibre général. Dans la mesure où il ne revient plus sur la question de l'égalité du nombre d'équations et du nombre d'inconnues dans le cadre de sa théorie de l'équilibre temporaire, on peut considérer qu'il suppose toujours l'existence d'un équilibre. Ainsi, ce qu'il appelle instabilité est la possibilité qu'un lundi donné le processus devant conduire au vecteur des prix d'équilibre ne converge pas.

L'analyse hicksienne souffre bien sûr de défauts qui sont aujourd'hui connus. La méthode employée dans Valeur et capital pour approcher la question de la stabilité est inadéquate (Samuelson, 1941). Mais surtout, comme Grandmont $(1975,1983)$ l'a montré, la question n'est pas tant celle de la stabilité que celle de l'existence de l'équilibre temporaire. Ceci étant, les travaux de Grandmont renforcent plus qu'ils ne l'affaiblissent le problème pointé par Hicks. Quant à l'analyse des conditions de stabilité de l'équilibre statique, pour Bliss, les conclusions de Hicks ne sont pas loin des conclusions qui se sont imposées par la suite : "The basic ideas fall out of the pages of Value and Capital » (1994 : 95).

Notons pour finir qu'à travers son analyse des conditions d'instabilité de l'équilibre temporaire, Hicks entend aller plus loin que son article de 1935 et que Keynes lui-même. Comme il le souligne dans Valeur et capital, l'hypothèse d'élasticité unitaire est celle que les économistes admettent implicitement. C'est l'hypothèse orthodoxe en matière d'anticipations de prix. Dans sa «suggestion » (1935), Hicks devait s'écarter de cette hypothèse pour faire pièce à l'optimisme des tenants de la théorie quantitative. Il s'appuyait en effet implicitement sur une hypothèse d'élasticité des anticipations de prix supérieure à l'unité : la hausse des prix déclenchait une chute de la demande de monnaie, ce qui supposait un effet de substitution intertemporelle. Dans Valeur et capital, Hicks porte son attaque sur le terrain des orthodoxes. Il montre que l'hypothèse de «la majorité des économistes » en matière d'anticipations de prix suffit à générer un scénario d'instabilité, si on tient compte des problèmes que pose l'ajustement à la baisse du taux d'intérêt. En un sens, cela va plus loin que le chapitre 19 de la Théorie générale dans lequel Keynes mobilise un effet de substitution intertemporelle

\footnotetext{
8 «It becomes very easily conceivable that downward adjustments may be necessary on a scale which would involve a negative rate of interest, if interest changes are to restore equilibrium. Consequently, the system may very easily be absolutely unstable » $(1939: 260)$. Pour une présentation plus détaillée de l'approche hicksienne de la trappe à liquidité voir Boianovsky (2004).
} 
déstabilisant et suppose donc implicitement une élasticité des anticipations de prix supérieure à l'unitế.

\subsection{La problématique des stabilisateurs et le rejet de la solution keynésienne}

Pour importante qu'elle soit aux yeux de Hicks, la «proposition » d'instabilité ne peut suffire à l'élaboration d'une théorie pure du capitalisme. Pour lui, si le capitalisme est instable, en temps normaux, son instabilité ne se manifeste pas sous la forme radicale d'une incapacité à atteindre l'équilibre. Nous retrouvons à ce stade la difficulté soulevée dans l'article de 1935. Mais en 1939, Hicks dispose désormais de ce qu'il considère être la solution keynésienne. Nous allons voir comment il la présente et pourquoi il la rejette.

\subsubsection{Le problème de l'articulation entre stabilité et instabilité}

Commençons par préciser la nature du problème auquel Hicks fait face en 1939. En développant l'intuition de son article de 1935, Hicks se retrouve face à une contradiction. Il est persuadé que l'instabilité potentielle de l'équilibre temporaire explique l'instabilité observée dans la réalité. Mais il est tout aussi persuadé qu'un modèle du système capitaliste acceptable doit être caractérisé par un équilibre temporaire stable.

Hicks affirme nettement que son modèle à équilibre temporaire instable est pertinent pour comprendre la réalité. Il écrit par exemple que « dans l'instabilité du modèle, on reconnaît l'instabilité que nous semblons détecter dans le système de la réalité » (1939: 258). Mais il affirme tout aussi nettement que ce modèle ne peut pas fournir une représentation générale du système capitaliste. Il est deux raisons à cela. En premier lieu, la stabilité de l'équilibre temporaire est une condition de réalisme ${ }^{10}$. En effet, Hicks considère que l'instabilité de l'équilibre temporaire en un point du temps signifie la «mort» de l'économie. Pour désigner cette situation, il emploie généralement le terme de «break-down » ou effondrement. Mais l'effondrement d'une économie capitaliste est un phénomène qui ne se produit pas «normalement» (1939: 255). Le plus souvent, l'instabilité se manifeste uniquement sous la forme de fluctuations de l'activité et le système fonctionne. En second lieu, la stabilité est une nécessité interne de la méthode développée dans les deux dernières parties de Valeur et capital. L'objectif de la «méthode de l'équilibre temporaire » est l'analyse de la dynamique de l'équilibre conçue comme une séquence d'équilibres. Ceci suppose évidemment que l'équilibre soit atteint chaque lundi. Cette hypothèse de travail est introduite par Hicks dans le chapitre 9 de l'ouvrage: «Nous supposons que le système économique est toujours à l'équilibre $\gg(1939: 131)$.

Cette contradiction offre selon moi la clé des derniers chapitres de Valeur et capital. Pour lui échapper, Hicks se lance à la recherche d'une articulation entre stabilité et instabilité. En d'autres termes, il forme le projet d'un modèle de cycle, c'est-à-dire un modèle traduisant l'instabilité sous sa forme réaliste, mais qui intègre aussi, d'une façon ou d'une autre, la possibilité de l'instabilité sous sa forme plus radicale. L'articulation devait être rendue possible par l'introduction de «stabilisateurs » dans le modèle initial :

"Consequently, somewhere among the modifications which would have to be introduced if our model is to be made realistic, we should expect to find possible

\footnotetext{
${ }^{9}$ «If, on the other hand, the reduction leads to the expectation, or even to the serious possibility, of a further wage-reduction in prospect, it will have precisely the opposite effect. For it will diminish the marginal efficiency of capital and will lead to postponement both of investment and of consumption » (1936: 263).

${ }^{10}$ «We are now in a position of having constructed a model economy, which we have found to lie upon the verge of instability. It is not a realistic model » (1939: 10).
} 
stabilizers -elements which limits the fluctuations of the economy, though they do not prevent it from fluctuating altogether » (1939: 258).

\subsubsection{La solution keynésienne}

Pour résoudre le problème initial du réalisme-irréalisme de l'instabilité, Hicks parait d'abord emmener son lecteur vers une solution keynésienne. Après avoir montré que l'ajustement du taux d'intérêt ne rétablissait pas l'équilibre en cas de déflation, il soutient que la stabilisation de l'économie est assurée par la rigidité des salaires. Ainsi modifié, le modèle de départ permet d'articuler le danger de l'instabilité à une représentation «réaliste » de l'économie. Si les salaires sont laissés flexibles, l'équilibre est instable. Avec des salaires rigides, l'équilibre temporaire est stable et le système peut rendre compte des fluctuations de la production et du chômage le long du sentier qu'il parcourt de semaine en semaine :

"The only reliable check within the system is the rigidity of wage-rates; though the operation of this check to downward instability is necessarily attended by a contraction of total output below the maximum technically possible and by the existence of unemployed labour $\gg(1939: 269)$.

Ce bouclage du modèle initial est présenté par Hicks comme la solution de Keynes :

"Mr. Keynes goes so far as to make the rigidity of wage-rates the corner stone of his system » (1939: 266).

$\mathrm{Ou}$, un peu plus loin:

"Unemployment is the best stabilizer we have yet found.

8. This is a profoundly distressing conclusion, yet it does not seem to be avoidable, so long as we assume unity elasticities of expectations. It is, of course, the conclusion of Mr. Keynes, who stresses it so much as to make his General Theory a General Theory of Employment » (1939: 269).

L'hypothèse de rigidité des salaires, combinée à l'hypothèse d'élasticité unitaire des anticipations de prix, serait donc la «pierre angulaire » de la théorie de Keynes ${ }^{11}$.

Le modèle keynésien de Valeur et capital correspond à la «solution intermédiaire » souhaitée par Hicks dans la «Suggestion». Dans ce modèle, comme dans la Théorie générale, l'instabilité ne se manifeste jamais sous la forme d'un «effondrement » au sens que Hicks prête à ce terme.

\subsection{3. «A very special case »}

Au dernier moment, Hicks écarte néanmoins la solution keynésienne. Trois pages avant la fin du chapitre 21 , deux critiques surgissent qui viennent remettre en cause le modèle à salaire rigide et élasticité unitaire des anticipations de prix.

La critique principale porte sur l'hypothèse d'élasticité unitaire des anticipations. Hicks écrit qu'une telle hypothèse implique un comportement très spécial. En effet, les agents considèrent toute variation des prix comme étant permanente. L'économiste illustre l'absurdité d'un tel comportement en prenant le cas particulier des anticipations statiques $\left(\mathrm{p}_{\mathrm{t}+\mathrm{i}}{ }^{\mathrm{a}}\right.$ $=\mathrm{p}_{\mathrm{t}}$ ). Dans ce cas, à chaque changement des prix courants, les agents se persuadent que les nouveaux prix seront éternels (1939 : 270). En d'autres termes, ils ignorent l'évolution passée des prix et sont susceptibles de commettre des erreurs systématiques. Hicks ajoute qu'un comportement caractérisé par une élasticité unitaire des anticipations ne peut pas être fréquent

\footnotetext{
${ }^{11}$ Remarquons la différence entre cette interprétation de la Théorie générale et celle que Hicks défend dans «Mr. Keynes and the Classics » en 1937. Dans l'article de 1937, en effet, Hicks présente la rigidité des salaires comme une hypothèse partagée par les Classiques et ne dit rien des anticipations dans l'approche de Keynes.
} 
dans la mesure où sa conséquence, l'instabilité de l'équilibre temporaire, s'observe rarement. L'hypothèse d'élasticité unitaire relève du cas particulier. Mieux vaut adopter comme point de départ une hypothèse d'anticipation de prix inélastique ou l'hypothèse selon laquelle les agents croient en l'existence d'un « niveau des prix normal ».

Dans un deuxième temps, Hicks affirme que l'hypothèse de rigidité des salaires serait incompatible avec l'hypothèse d'élasticité unitaire. Son raisonnement peut être reconstruit de la façon suivante. Le salaire réel fait l'objet d'une négociation qui est fonction de critères relevant de l'équité ( fairness»). La rigidité des salaires est donc d'abord réelle. Hicks suppose ensuite que les agents concernés ajustent les salaires nominaux de façon à préserver le salaire réel «juste». Si les anticipations sont élastiques, les agents considèrent que toute modification courante des prix est permanente. Ils réviseront alors les salaires à chaque variation des prix. Les salaires doivent être supposés flexibles. L'argument paraît solide. A l'opposé, si les anticipations sont inélastiques, les agents «fixent le taux de salaire monétaire à un niveau qui leur paraît juste compte tenu du niveau des prix normal » (1939: 270). Ce n'est que dans ce cas que les salaires pourraient être supposés rigides. Cette seconde partie de l'argument est plus fragile. Nous y reviendrons plus loin.

Contentons-nous d'un rapide bilan de l'argumentation. Hicks offre deux raisons convaincantes de renoncer à la combinaison d'une hypothèse d'élasticité unitaire des anticipations et d'une hypothèse de rigidité des salaires. Cette critique le conduit à mettre en avant un modèle combinant inélasticité des anticipations et rigidité des salaires. Mais si ce modèle peut rendre compte des fluctuations de l'emploi, il exclut totalement la possibilité d'une instabilité de l'équilibre en un point du temps.

\section{3 « Vie et mort de l'ensemble du système » et théorie du cycle}

"It is one of the most exciting characteristics of the method of analysis we are pursuing in this book that it enables us to pass over, with scarcely any transition, from the little problems involved in detailed study of the behaviour of a single firm, or single individual, to the great issues of the prosperity or adversity, even life or death, of whole economic system » (1939: 245).

Dans les deux dernières pages du chapitre 21 de Valeur et capital, Hicks esquisse «the assumptions which ought to be made in order to get a reasonably realistic model of the economic system » (1939: 271). Ce qu'il propose est une nouvelle façon d'articuler stabilité et instabilité à l'intérieur de son modèle d'équilibre temporaire.

\subsubsection{Vers une endogénéisation de l'élasticité des anticipations}

La clé du modèle envisagé à ce stade est l'endogénéisation de l'élasticité des anticipations de prix des agents. Hicks adopte comme point de départ l'hypothèse d'une élasticité des anticipations agrégée nulle ou telle que le système soit stable. Mais l'évolution de l'économie, les chocs auxquels elle est soumise, peuvent faire varier cette élasticité au point de rendre le système instable.

Hicks introduit cette modification sous la forme d'une hypothèse d'hétérogénéité des agents composant l'économie. Y cohabitent désormais des agents «sensibles », aux anticipations élastiques, et des agents « insensibles », aux anticipations inélastiques :

"There is no reason to suppose that all price-expectations are inelastic. Indeed, we should do better to assume a good deal of variation in different people's elasticities of expectations» (1939: 271). 
La présence des agents «sensibles » introduit une instabilité potentielle dans le système. Une économie soumise à des « perturbations violentes » peut voir leur proportion augmenter à l'intérieur de la population :

"We have to be prepared do deal with a range of possible cases, varying from that of a settled community, which has been accustomed to steady conditions in the past (and which for that very reason, is not easily disturbed in the present), to that of a community which has been exposed to violent disturbances of prices (and which may have to be regarded, in consequence, as being economically neurotic)» (1939: 272).

Hicks a précisé un peu plus tôt qu'un accroissement de l'élasticité des anticipations de prix implique un retour à la flexibilité des salaires. Un tel accroissement fait donc basculer l'économie dans l'instabilité :

"Once they become convinced that these changes [in related prices] are permanent changes, there is a tendency for wages to change ; in situations of extreme instability, when they have lost their sense of normal prices, negotiators have recourses to automatic sliding scales and the rigidity of money wages ceases altogether." (1939: 270-1)

Ce que Hicks introduit à ce stade est un schéma dans lequel l'élasticité des anticipations serait endogène et commanderait la stabilité du système économique. A la « pierre angulaire » keynésienne de la rigidité des salaires, il substitue la pierre angulaire hicksienne constituée par l'élasticité variable des anticipations de prix.

\subsubsection{Intégration du scénario d'instabilité à la théorie des cycles}

Hicks reprend ces ingrédients et précise son projet dans la conclusion de Valeur et capital consacrée à des « réflexions provisoires » sur le cycle des affaires. Les bases de l'analyse sont posées dans le chapitre 23. Hicks avance l'existence d'un décalage temporel entre les accroissements de revenu et les accroissements de production générés par l'accumulation du capital. Le boom est déclenché par une augmentation exogène de l'investissement. Ceci génère un accroissement des revenus plus rapide que l'accroissement de la production et par conséquence un excès de demande qui stimule le boom. Mais lorsque les capacités de production nouvelles deviennent disponibles, les revenus anticipés ont déjà été distribués et la demande manque. L'excès d'offre et la déflation succède à la montée des prix. A l'intérieur de ce schéma, Hicks introduit le partage de la population entre agents sensibles et agents insensibles et donne un rôle déterminant à l'élasticité des anticipations globale de la communauté. Dans cette perspective, le boom est nourrit par le basculement progressif de la population dans le groupe des agents «sensibles», ceci permettant un accroissement permanent de la demande. Un agent qui devient sensible anticipe une hausse future des prix, ce qui déclenche un effet de substitution intertemporelle: ses demandes courantes augmentent. La récession peut avoir deux causes principales. La première est l'existence d'un noyau dur d'agents «insensibles » qui vient enrayer la progression de l'activité économique. Ce problème peut se manifester sous la forme d'une restriction de l'accès au crédit dans la mesure où les banques observent un écart croissant entre la monnaie utilisée par les agents sensibles et la monnaie des agents insensibles (1939: 296-7). La seconde cause de retournement du cycle est « the achievement of the capital accumulation planned in the first stage and now carried out» (1939: 297). Dans les deux cas, l'économie affronte une récession dont la dynamique est fonction de la foi des agents en une valeur normale des prix et des salaires. L'existence de cette valeur normale stabilise l'économie. Mais, comme dans le chapitre 12 de la Théorie générale, la norme est fragile : 
«But in spite of that we must be careful not to put to much trust in these factors of stability, not to suppose that they can permanently save the situation. (...). But if nothing happens to induce a genuine resumption of the process of accumulation, then the stabilizing factors are bound to grow weaker as time goes on. Prolonged experience of low prices will disturb the norms, and induce a further revision of expectations downwards. A secondary slump will set in, far more dangerous than the first, since there is less resistance available to prevent collapse » (1939: 298).

Si on relie ce passage à la fin du chapitre 21 , la conclusion se dessine nettement. Durant la récession, l'inélasticité des anticipations stabilise l'économie. Cette inélasticité implique des effets de substitution intertemporelle stabilisant et, surtout, la rigidité des salaires. Mais ces anticipations stabilisantes ne suffisent pas à provoquer le rebond. Et en l'absence d'un choc positif («something new», 1939: 298) qui face redémarrer l'économie, la croyance des agents en la valeur normale des prix et des salaires peut s'effondrer. Leurs anticipations peuvent alors devenir élastiques, les salaires flexibles, et l'économie basculer dans l'instabilité.

Dans les derniers paragraphes de la conclusion, Hicks renforce le ton catastrophiste de sa représentation du cycle. Le danger d'un effondrement de l'économie dépend des facteurs qui ont déclenché la récession. Il est à son maximum lorsque la récession est due à un épuisement des opportunités d'investissement. Dans ce cas, le rebond est suspendu à un regain de l'innovation. Or, d'après Hicks, il y a des raisons de penser que la vague d'innovations associée à la révolution industrielle est épuisée (1939 : 301-2). En d'autres termes, le Hicks de 1939, en phase avec son époque, s’interroge sur la capacité de survie du capitalisme.

\subsubsection{Garder en mémoire le caractère mortel du système économique}

Nous voyons maintenant se dessiner les grandes lignes du modèle projeté par Hicks dans les derniers chapitres de Valeur et capital.

Dans les modèles keynésiens de Hicks (celui de 1939 comme celui de 1937), l'instabilité ne se déclare jamais mais on peut vérifier théoriquement qu'une baisse des salaires ne ramènerait pas le plein emploi. Dans le modèle hicksien, l'inélasticité des anticipations, autrement dit la foi en la stabilité des prix, garantit, en temps normal, la stabilité du système. Mais si cette foi est remise en cause en période de récession, l'économie est susceptible de basculer dans l'instabilité. A ce stade, l'instabilité de l'équilibre en un point du temps n'est plus opposée à une représentation de l'instabilité en termes de cycles, comme dans l'article de 1935. Ce danger est introduit à l'intérieur de la théorie des cycles. Le modèle projeté possède ainsi deux caractéristiques distinctives au-delà de la méthode de l'équilibre temporaire. La première est le rôle de stabilisateur ultime accordé aux anticipations. La seconde est la prise en compte de la possibilité que le système économique s'effondre. A la différence de Keynes, Hicks considère qu'une situation où se combinent la trappe à liquidité et des anticipations de prix élastiques ne peut pas être un équilibre de sous-emploi. En effet, dans ces conditions, les salaires sont flexibles. Quand Keynes se contente de pointer l'absence de retour automatique au plein emploi ou le danger de dégradation progressive de la situation, Hicks met en avant le risque pour lui bien réel d'un effondrement du système ${ }^{12}$.

\section{Pourquoi Hicks a délaissé le projet de 1939}

Dès 1950, Hicks a abandonné la piste esquissée dans les derniers chapitres de Valeur et capital. Dans A Contribution to the Theory of the Trade Cycle (1950), il développe bien une

\footnotetext{
${ }^{12}$ Keynes envisage lui aussi l'effondrement du système capitaliste dans le chapitre 19 de la Théorie générale (1936: 303-4) mais uniquement comme un cas d'école, à la manière de Hicks en 1935.
} 
théorie des cycles. Mais il écarte le cadre walrassien au profit du modèle IS-LM. Les anticipations n'interviennent plus que de façon implicite et le scénario d'un effondrement de l'économie disparaît. Nous n'entrerons pas dans une étude détaillée de la transition de l'ouvrage de 1939 à celui de 1950. L'objectif de cette dernière partie est de faire apparaitre les difficultés que posent, dès 1939, l'élaboration des trois ingrédients clés du modèle esquissé par Hicks : le lien entre rigidité des salaires et élasticité des anticipations, la théorie des anticipations et la prise en compte de l'instabilité. Ces difficultés nous semblent suffisantes pour comprendre pourquoi Hicks est revenu à la solution intermédiaire de 1935 sous la forme d'un cycle contenu par un plancher et un plafond ${ }^{13}$.

\subsection{La question des fondements de la rigidité des salaires}

Pour écarter l'instabilité dans le cas général, Hicks introduit la rigidité des salaires. Cela pose deux problèmes. Pour conserver la possibilité de l'instabilité, il faut que les salaires puissent redevenir flexibles. Hicks résout ce problème en introduisant un lien entre rigidité des salaires et élasticité des anticipations. Toute son analyse est donc suspendue à la justification de ce lien, justification qui est en réalité un point faible de sa construction. De façon plus générale, le phénomène de la rigidité des salaires entre en conflit avec la méthode défendue dans Valeur et capital.

Hicks présente les configurations inélasticité-rigidité et élasticité-flexibilité comme si elle était symétrique. Mais il est plus facile de justifier la flexibilité des salaires que leur rigidité lorsqu'on raisonne à partir d'un cadre walrassien.

Le raisonnement qui conduit Hicks à rejeter l'association entre élasticité des anticipations et rigidité des salaires nous semble convaincant. Son défaut est d'être trivial. Si l'on suppose, en bon walrassien, que les agents n'ont pas de vision d'ensemble du système et si l'on suppose par ailleurs, de façon moins walrassienne, qu'ils cherchent à préserver leur salaire réel, il est logique de conclure que les salariés doivent accepter une réduction de leurs salaires monétaires en phase de déflation. On peut tout de même reprocher à Hicks d'ignorer deux arguments importants de Keynes. Le premier, présenté dans le chapitre 2 de la Théorie générale, est que les salariés défendent leur salaire réel relatif dans un contexte où la négociation sur les salaires monétaires est décentralisée (Hoover, 1995). Le second argument revient à supposer que les agents ont une vue d'ensemble de l'économie et perçoivent le danger que représente la baisse des salaires pour la stabilité et l'emploi. Il est alors rationnel pour eux de préserver le niveau des salaires face à la déflation (Benetti, 1998).

La justification apportée à la rigidité des salaires est plus problématique. A supposer que les agents cherchent à préserver un salaire réel jugé «juste » (cf. supra section 2.2.3), on ne comprend pas pourquoi ils n'ajusteraient pas les salaires face à des variations de prix considérées comme transitoires. La rationalité de cette institution est d'autant moins évidente que le système est stable lorsque les anticipations de prix sont inélastiques. En d'autres termes, laisser les salaires s'ajuster garantirait le plein emploi. Du point de vue des seuls salariés, la fixation des salaires revient à accepter des pertes de revenus en phase de hausse des prix et le chômage en phase de déflation.

Il nous semble qu'à ce stade de l'exposé, Hicks cherche à concilier l'introduction de l'hypothèse de rigidité des salaires avec la méthode « d'analyse logique pure du capitalisme » affichée dans l'introduction de l'ouvrage :

\footnotetext{
${ }^{13}$ Nous écartons l'explication qui s'appuierait sur l'omission de l'effet d'encaisse réelle dans Valeur et capital et sur l'importance que cet argument prend dans les débats sur le message de Keynes au cours des années 1940 et 1950. En effet, si Hicks reconnaît la validité théorique de l'effet Pigou, il en minimise la portée pratique et soutient qu'il ne suffit pas à assurer la stabilité de l'économie dès lors que des effets revenus asymétriques (effets de répartition) interviennent (1939: 334-5).
} 
« This is a work on Theoretical Economics, considered as the logical analysis of an economic system of private enterprise, without any inclusion of reference to institutional controls. I shall interpret this limitation pretty severely. For I consider the pure logical analysis of capitalism to be a task in itself, while the survey of economic institutions is best carried on by other methods, such as those of the economic historian (even when the institutions are contemporary institutions) » (1939: 7).

Ces considérations méthodologiques précisent une critique adressée à Keynes. D’après Hicks, ce dernier confondrait les registres de l'analyse logique et de l'histoire économique. En conséquence, son lecteur peinerait à démêler dans son travail «les fruits de la pure logique » et les «fruits de son propre point de vue sur les questions sociales » (1939: 4). Hicks précise encore son approche en indiquant qu'elle suppose la concurrence parfaite et en affirmant que l'hypothèse inverse aurait des «conséquences destructrices pour la théorie économiques » (1939: 83).

Hicks entend donc inscrire le problème de l'instabilité du système de marché dans le cadre de l'analyse logique pure. Mais l'instabilité appelle l'hypothèse de rigidité des salaires et cette dernière relève à l'évidence de l'histoire économique. En effet, il invoque d'abord trois explications possibles de ce phénomène : le "contrôle législatif », «l'action des monopoles » et une «notion de juste prix ${ }^{14}$. La rigidité des salaires implique à la fois des institutions inscrites dans l'histoire et la concurrence imparfaite. En reliant la rigidité à l'inélasticité des anticipations, Hicks semble vouloir contourner cette difficulté. Il ouvre alors la boîte de Pandore des fondements microéconomiques de la rigidité : "Pourquoi les taux de salaire seraient-ils rigides ?»(p. 270). Alors qu'il se contente d'évoquer comme en passant les causes de rigidité un peu plus haut dans le chapitre, il entend désormais faire de ce phénomène la conséquence d'un choix rationnel de la part des agents.

Mais son argumentation fait appel à la notion de «juste prix » afin d'expliquer la détermination du salaire réel. En arrière plan, on peut discerner de surcroît un processus de négociation qui relève de la concurrence imparfaite. Notons que cette mobilisation de la notion de «fairness » contredit l'analyse développée dans The Theory of Wages (1932) où Hicks présente les notions d'éthique comme un habillage du jeu de l'offre et de la demande qui seul détermine les salaires en concurrence parfaite ${ }^{15}$. Dans Valeur et capital, l'éthique interfère avec le jeu du marché.

Hicks essaie de rendre compte de la rigidité des salaires à l'intérieur du cadre de la théorie pure, c'est-à-dire comme une caractéristique endogène du système capitaliste. Mais cette tentative nous semble peu concluante. On peut imaginer qu'il ait eu conscience de relâcher la discipline méthodologique affichée dans l'introduction de son ouvrage. La difficulté posée par la rigidité des salaires constitue probablement le point de départ de la réflexion qui l'a conduit à défendre la méthode des prix fixes dans Capital and Growth (1965) et a certainement joué un rôle déterminant dans l'abandon de la méthode d'équilibre temporaire. Cependant, rien n'interdit de revenir sur des déclarations de méthode trop radicales. C'est d'ailleurs ce qu'ont fini par faire les macroéconomistes contemporains en introduisant des facteurs de rigidités nominales exogènes dans leurs modèles de cycle. De ce point de vue, on peut accorder à Hicks le mérite de poser le problème d'une façon originale en tentant de relier la rigidité à l'état des anticipations.

\footnotetext{
14 "This rigidity [of prices] may be due to legislative control, or to monopolistic action (of the sleepy sort which does not strain after every gnat of profit, but prefers a quiet life). It may be due to lingering notions of a 'just price'. The most important class of prices subject to such rigidities are wages rates; they are affected by rigidity from all three causes. They are particularly likely to be affected by ethical notions, since the wagecontract is very much a personal contract, and will only proceed smoothly if it is regarded as 'fair' by both parties" (1939: 265).

15 "These rules of fairness and justice are simply rough-and-ready guides whereby the working of supply and demand is anticipated. That they are not perfect guide is shown by the fact that they are so often broken." (Hicks, 1932: 80)
} 


\subsection{Une théorie avortée de la formation des anticipations}

Dans sa recension de A Contribution to the Theory of the Trade Cycle, Kaldor (1951) s'étonne du silence de l'auteur sur la question des anticipations ${ }^{16}$. En effet, le thème des anticipations est l'un des fils rouges de la réflexion de Hicks durant les années 1930. Le but de cette section est d'éclairer les raisons pour lesquelles il l'a délaissé. Nous ferons d'abord apparaître le contraste entre la nécessité criante d'une théorie de la formation des anticipations à l'intérieur de la construction hicksienne et le caractère pour le moins embryonnaire de ce qui est proposé dans Valeur et capital. Nous discuterons ensuite les raisons pour lesquelles Hicks offre un traitement si peu systématique de cette dimension de l'analyse.

\subsubsection{Théoriser la formation des anticipations : une nécessité interne}

L'absence de théorie concernant la formation des anticipations et la nécessité de combler cette lacune est déjà formulée dans «A Suggestion for Simplifying the Theory of Money » (1935). D'après Hicks, la demande de monnaie dépend des coûts de transaction, du rendement des titres et de la fréquence des paiements. Les deux derniers facteurs doivent être anticipés. Cela pose le problème de la formation de ces anticipations :

"If I am right, the whole problem of applying monetary theory is largely one of deducing changes in anticipations from the changes in objective data which call them forth» (1935: 13).

Hicks insiste ensuite sur la difficulté que pose ce passage des données objectives aux anticipations. Il parle à ce sujet d'un «fossé qui gît sous nos pieds » [ «a gap which lies behind us »] (1935 : 14). Lorsqu'il en vient à évoquer la possibilité d'un équilibre instable, dans le même article, Hicks souligne bien qu'il recourt à une hypothèse particulière concernant la formation des anticipations et qu'ainsi il contourne l'obstacle (« this is jumping over my gap », 1935: 16). On peut considérer que cette remarque revient à affirmer la nécessité d'une solution plus adéquate.

Ce problème est à nouveau évoqué dans la recension de la Théorie générale publiée par Hicks en 1936. Ce dernier souligne alors la parenté entre l'approche de Keynes et celle qu'il développe en s'inspirant des Suédois. Le trait qui retient son attention est l'intégration des anticipations comme une donnée de l'analyse au même titre que les dotations et les préférences des agents (1936: 240). Ceci permet d'analyser les propriétés de statique comparative d'un système en dehors de l'équilibre stationnaire ou de l'équilibre normal. Mais cela ne permet pas l'étude des effets d'une perturbation de l'équilibre au-delà de la période courante. Une théorie de la formation des anticipations fait défaut.

Ainsi, dès le milieu des années 1930, Hicks pointe explicitement une lacune de la «théorie monétaire » : le «gap » de la formation des anticipations.

Comme nous l'avons vu plus haut, ce problème resurgit dans les derniers chapitres de Valeur et capital. Hicks entend inscrire le danger de l'instabilité à l'intérieur d'une théorie des cycles conçue comme l'analyse d'une séquence d'équilibres temporaires. Or, il se donne comme hypothèses de base des anticipations de prix inélastiques et la rigidité des salaires, des hypothèses qui, d'après lui, excluent le scénario d'un équilibre instable. Pour justifier la possibilité d'un tel scénario, il faut donc expliquer comment l'élasticité des anticipations de prix peut varier d'une semaine à l'autre en passant de 0 à 1 ou plus. En outre, Hicks suppose que le degré d'élasticité des anticipations commande la rigidité des salaires. Il substitue à la «pierre angulaire » de la théorie de Keynes, une «pierre angulaire » hicksienne : l'élasticité

\footnotetext{
16 "On the subject of expectations, to which Mr. Hicks made such distinguished contributions in the past, the present work is remarkably silent” (Kaldor, 1951 : 839 et 846). Voir aussi Coddington (1983: 86-7).
} 
des anticipations et la théorie qui rendrait compte de ses variations au cours du cycle. Endogénéiser l'élasticité des anticipations est bien une nécessité interne de l'approche invoquée à la fin de l'ouvrage de 1939.

\subsubsection{Hicks a-t-il cherché à théoriser les variations de $e$ dans Valeur et capital ?}

Le signe le plus évident d'un effort d'élaboration théorique concernant la formation des anticipations dans Valeur et capital est le concept d'élasticité des anticipations de prix. Ce concept n'a pas seulement la vertu de simplifier l'analyse, il débouche aussi sur une endogénéisation des anticipations de prix que l'on pourrait qualifier d'intra-temporelle.

Toute l'analyse hicksienne est sous-tendue par une même théorie des choix intertemporels. Les agents doivent établir des plans pour le présent et pour toutes les périodes futures à l'intérieur de leur horizon temporel. Ces plans dépendent des prix présents et des prix futurs anticipés. L'enjeu essentiel de cette théorie est l'analyse des effets de substitution intertemporels générés par les variations du rapport entre prix présents et prix futurs. Au regard de la théorie des choix statiques, un nouveau problème surgit: l'état présent de l'économie peut avoir un effet sur les prix anticipés des biens futurs. Or, «si notre théorie doit conduire à des résultats utiles, nous devons prendre en compte cet effet » (1939: 204). Le concept d'élasticité des anticipations de prix relève d'une méthode pour rendre la discussion la plus simple possible. Hicks suppose que le rapport entre prix futurs et prix courants est réductible à une seule grandeur. Ceci revient à supposer que le prix futur anticipé d'une marchandise dépend uniquement du prix courant de cette marchandise et que la relation entre le prix courant et le prix futur est la même (élasticité identique) quelque soit l'horizon du prix et quelque soit l'agent considéré. Hicks considère que son hypothèse est la plus générale car elle n'implique aucune distinction entre les agents ou entre les prix (salaires et prix des biens en particulier). Le concept de Hicks n'introduit pas seulement une simplification. Il endogénéise les prix anticipés puisqu'ils sont désormais fonction des prix courants. On peut traduire l'idée en écrivant: $P_{t+T}^{a}=A P_{t}^{e}$ où $e$ est l'élasticité des anticipations ${ }^{17}$. L'endogénéisation reste néanmoins partielle car l'élasticité, la clé de l'analyse de stabilité de l'équilibre temporaire, demeure un paramètre.

Cette approche est suffisante pour montrer la possibilité théorique d'une situation d'instabilité. Mais il faut encore convaincre le lecteur de la plausibilité d'une telle situation. Pour cela, il faut expliquer pourquoi l'élasticité des anticipations est susceptible d'atteindre voire de dépasser l'unité en phase de récession. En d'autres termes, le concept d'élasticité des anticipations permet de saisir l'effet d'une variation des prix courants sur les prix anticipés à l'intérieur la période courante. Mais il ne donne pas d'indication sur la manière dont la situation courante de l'économie peut affecter la formation des anticipations lors des périodes ultérieures. Il reste à opérer l'endogénéisation intertemporelle des anticipations.

Hicks a-t-il essayé de théoriser les variations de $e$ ? Cela n'est pas certain. Dans le contexte de Valeur et capital, la théorisation serait une tentative d'expliquer les variations de l'élasticité des anticipations en lien avec les concepts de la théorie des choix intertemporels développée par l'économiste. Hicks propose bien une série d'arguments justifiant les variations de $e$ mais il s'agit de fragments d'analyse éparpillés et incomplets.

\footnotetext{
${ }^{17}$ Pour saisir l'apport de Hicks, on peut comparer son approche à celle de la Théorie générale. Tant qu'il s'intéresse à la détermination de l'équilibre à l'intérieur d'une période donnée, Keynes considère que l'état des anticipations à long terme est donné. Les variations de l'emploi et des prix n'ont pas d'effet sur les grandeurs anticipées. C'est uniquement dans le chapitre 19, lorsqu'il semble étudier une séquence d'équilibres, qu'il tient compte de l'effet de la variation des prix courants sur les anticipations de la période suivante. Mais l'analyse est très informelle.
} 
Hicks considère que l'agent économique croit généralement en l'existence de «prix normaux ». Si les prix s'écartent de leur valeur normale, ils doivent y revenir. L'hypothèse de base est donc celle de l'inélasticité des anticipations de prix. Le problème consiste à expliquer comment les anticipations de prix deviennent élastiques. Nous avons vu comment, à la toute fin du chapitre 21 et dans la conclusion de Valeur et capital, Hicks relâche l'hypothèse d'uniformité des anticipations d'un agent à l'autre et étudie comment une proportion croissante de la population peut basculer dans le camp des agents à anticipations élastiques. D'un chapitre à l'autre, on peut repérer quatre types d'arguments invoqués pour expliquer comment l'élasticité des anticipations de prix d'un agent change au cours du cycle.

i) Premier argument, dans la phase ascendante du cycle, les agents développent des anticipations élastiques parce que les prix des marchandises qu'ils vendent, où qu'ils achètent s'élèvent. C'est le cas des industriels avant d'être celui des salariés ou de leurs syndicats :

"At the same time there is a tendency to a general rise in the prices of the more sensitive commodities; and within a little, we may suppose that some industrialists may have time to develop elastic expectations (at least for that length of time which is mainly relevant for the sort of production processes in which they are engaged) » $(1939: 295$ 6).

La précision entre parenthèse fait écho à un argument développé, dans une note de bas de page du chapitre 21, pour expliquer l'hétérogénéité des anticipations (1946, note 1:271). Même s'ils sont persuadés que la hausse des prix est temporaire (foi en un prix normal), les industriels développent des anticipations élastiques dès lors qu'ils considèrent que la hausse va se maintenir voire se poursuivre jusqu'au terme de leur "processus de production », c'està-dire à l'intérieur de l'horizon de calcul pertinent pour prendre leurs décisions. Mais même une fois assemblés ces deux fragments d'analyse, l'argument paraît trop court. On voudrait comprendre comment les industriels en viennent à croire que la hausse des prix va persister à l'intérieur de leur horizon de planification. Un processus de révision des anticipations qui ferait dépendre $e$ des erreurs passées est-il à l'œuvre ? On aimerait aussi comprendre comment se détermine l'horizon de calcul. Cependant, l'argument prend bien appui sur la théorie des choix intertemporels et représente un embryon de théorisation.

ii) Deuxième argument, "l'optimisme se diffuse de lui-même à travers la communauté » (1939 : 296). C'est la notion cambridgienne de la «vague d'optimisme » reprise par Keynes dans la Théorie générale ${ }^{18}$. Dans les termes de son ouvrage, Hicks aurait pu traduire en écrivant que l'élasticité $e_{i}$ d'un individu i dépend de l'élasticité moyenne des agents. Mais pour compliquer les choses, il considère que cette contamination se fait graduellement. Il suppose donc que certains agents sont plus sensibles que d'autres à l'euphorie ambiante, certains ne l'étant pas du tout. Dans son article de 1935, il expliquait l'insensibilité de certains agents par leurs faibles revenus. Mais cette idée n'est pas reprise en 1939. L'idée pourrait se prêter à une formalisation simple. Hicks s'en tient cependant à un énoncé purement descriptif.

iii) Une explication majeure de l'élasticité des anticipations est la «perte du sens des prix normaux » (1939: 271). Les agents croient habituellement que les prix convergent vers leurs valeurs normales. Mais si l'expérience invalide cette croyance, la notion des prix normaux s'effondre. Cela peut-être la conséquence de «perturbations violentes des prix » $(1939: 272)$ ou au contraire de l'absence de choc exogène. En phase de récession, comme nous l'avons souligné plus haut, c'est l'absence d'innovation et par conséquent l'absence de rebond des prix qui conduit à des anticipations élastiques et, éventuellement à l'effondrement de l'économie : «Prolonged experience of low prices will disturb the norms, and induce a further revision of expectations downwards » (1939: 298). L'effondrement de la notion de prix normal évoque la précarité de l'évaluation conventionnelle dans le chapitre 12 de la Théorie

\footnotetext{
${ }^{18}$ Voir Bateman (2003) et Laidler (1999).
} 
générale. Il s'agit d'un point central pour Hicks dans la mesure où cet argument fonde la possibilité que la récession dégénère. Le problème sur lequel on bute à ce stade est le caractère sous-développé du concept de prix normal. Est-il une convention arbitraire ou reflète-t-il certains facteurs objectifs ? La notion de prix ou de salaire normal peut s'effriter mais le taux d'intérêt normal, lui, dépend de fondamentaux, coûts de transactions et facteurs de risque, que Hicks estime stables. Le processus de formation de la croyance en un prix normal ou la façon dont le prix normal s'insère dans une théorie des choix intertemporels n'est pas exploré de façon systématique.

iv) En phase de récession, la chute des prix nourrit la «psychologie de la dépression »:

"Now when prices are falling, a psychological condition of depression seems to be induced, which is very unpropitious to distant planning ${ }^{2} »(1939: 263)$.

Hicks (1939 : 125-6 et 225) propose d'intégrer ce facteur à l'analyse sous la forme d'une prime de risque («an allowance for risk ») qui viendrait amputer les prix anticipés. Plus la date considérée est éloignée du présent, plus le risque perçu par l'agent est important. Ce facteur détermine alors l'horizon de planification des agents. Si l'on adopte le point de vue du vendeur, son prix de vente décroît à mesure que l'horizon s'éloigne jusqu'à ce que l'activité ne soit plus rentable et que le calcul soit inutile. Hicks considère qu'une phase de récession correspond à une montée de l'incertitude. La prime de risque augmente et l'horizon des agents se raccourcit. Ceci rend leurs décisions moins sensibles aux variations du taux d'intérêt (1939 : 263). Mais si l'horizon de planification se raccourcit, Hicks affirme aussi que les anticipations relatives aux prix deviennent plus élastiques dans la mesure où le retour au prix normal à long terme est négligé. Les agents se focalisent sur le court terme. Bien qu'incomplet, l'argument relève d'une véritable élaboration théorique.

Lorsqu'il en vient à traiter de la formation des anticipations, la faiblesse de la théorisation est aussi frappante que la multiplication des arguments pour expliquer l'hétérogénéité et les variations de $e$. Il y a là une nécessité d'élaboration théorique à laquelle Hicks ne répond pas de façon rigoureuse et systématique.

\subsubsection{Un défi insurmontable?}

Pourquoi Hicks ne va-t-il pas plus loin dans sa tentative d'élaborer la théorie des anticipations ? Trois raisons peuvent être invoquées. Premièrement, Hicks se trouve face à une difficulté technique insurmontable au regard de ses compétences en économie mathématique et de l'état de développement de la théorie de l'équilibre général dans les années 1930. Deuxièmement, en adoptant le concept d'équilibre temporaire, Hicks rejette l'idée d'une convergence vers un équilibre normal. Comment alors justifier l'ancrage des anticipations dans la croyance en des prix normaux ? Troisièmement, il semble qu'il ait considéré que le problème de la formation des anticipations débordait le cadre de la théorie pure. Ce serait alors en toute connaissance de cause qu'il se serait contenté de remarques descriptives concernant les variations de $e$.

Le sous-développement de la théorie des anticipations hicksienne doit être resitué dans le contexte méthodologique de Valeur et capital. Nous retrouvons ici la distinction hicksienne, introduite dans la section précédente, entre «l'analyse logique pure du capitalisme » qui fait abstraction des institutions et l'histoire économique qui en rend compte. Dans le cadre de l'analyse logique, l'économiste doit déduire toute proposition concernant le comportement d'un groupe d'agents ou du système de marché dans son ensemble de la théorie des choix, voire de la théorie du consommateur : 
"What begins as an analysis of the consumer's choice among consumption goods ends as a theory of economic choice in general. We are in sight of a unifying principle of the whole economics $\gg(1939: 24)$.

Par ailleurs, toute proposition se doit d'être généralisée à $\mathrm{n}$ agents ou à $\mathrm{n}$ biens et cela suppose une démonstration mathématique. C'est ainsi que Hicks justifie l'appendice mathématique à la fin de son ouvrage :

"The purpose of this Appendix is not merely the transcription of the argument of the text into mathematical symbols; I see little advantage to be got from doing that. When the verbal (or geometrical) argument is conclusive, it gains nothing from being put in another form. What can be gained, however, is the assurance that our argument is completely general; that what has been proved in the text for two, or three, or four, commodities, is true for $n$ commodities. In this appendix I shall concentrate upon the proof of that 'generality'» (1939: 303).

Or, si Hicks esquisse une présentation du choix intertemporel du producteur il n'offre rien d'équivalent pour le consommateur et ne formalise pas son système d'équilibre temporaire. Dans ce contexte, il est peu étonnant qu'il n'ait pas élaboré plus à fond sa théorie des anticipations. La difficulté technique était telle qu'il a préféré opter pour une approche plus simple des questions macroéconomiques dans son ouvrage de 1950.

Un autre problème lié à la méthode défendue par Hicks peut être mis en avant. Dans sa recension de Keynes en 1936, Hicks insistait sur l'abandon par Keynes de l'analyse marshallienne du cycle en termes de déviation par rapport à une norme. En 1939, il revendique cet abandon pour lui-même. La séquence des équilibres temporaires n'a pas de raison de conduire à un équilibre supérieur. L'abandon de cette norme explique l'inconsistance de la notion de prix normal dans Valeur et capital. Cette inconsistance affaiblit bien sûr considérablement la théorie des anticipations de Hicks dont elle est un ingrédient essentiel.

Les textes publiés par Hicks au milieu des années 1930 laissent entrevoir une autre explication. Hicks y souligne la difficulté spécifique que pose l'élaboration d'une théorie des anticipations ou ce qu'il appelle en 1936 «l'inconnue psychologique ».

Il faut d'abord revenir sur l'article de 1935. Après avoir formulé la nécessité de déduire «les changements d'anticipations des changements de données », il se livre à une mise au point d'ordre méthodologique :

"Obviously, this is not an easy task, and, above all, it is not one which can be performed in a mechanical fashion. It needs judgment and knowledge of business psychology much more than sustained logical reasoning. The arm-chair economist will be bad at it, but he can at least begin to realise the necessity for it, and learn to cooperate with those who can do it better than he can » (1935: 13-4).

Pour Hicks, les ressources de l'analyse logique pure ou de la théorie des choix ne peuvent suffire lorsqu'il est question d'expliquer le lien entre «les données objectives » et les anticipations des agents. Il faut alors plus qu'un « raisonnement logique soutenu ». Mais cela ne signifie pas que ce problème échappe totalement à la théorie :

"When once the connection between objective facts and anticipations has been made, theory comes again into its rights; and it will not be able to complain of a lack of opportunities» (1935: 14).

L'économiste doit travailler avec le praticien ou l'économiste appliqué pour établir une hypothèse concernant le lien entre données et anticipations. Une fois cette hypothèse élaborée 
et explicitée, l'appareillage de l'équilibre général peut entrer en action. Ce que décrit ici Hicks, c'est la démarche qui fonde l'essentiel de sa théorie de l'équilibre temporaire en 1939. Une hypothèse est formulée sur le lien entre prix présent et prix anticipés à l'intérieur de la période courante, hypothèse résumée par le concept d'élasticité des anticipations. Puis l'analyse suit son cours. Mais il reste ce «gap which lies behind us » (1935: 14). L'hypothèse, portant sur les abîmes de la psychologie, reste une chose fragile.

Le propos de Hicks se fait plus pessimiste dans sa recension de la Théorie générale en 1936. Keynes montre la possibilité de traiter les anticipations comme un paramètre de l'équilibre en un point du temps. Mais il ne dit rien sur la façon dont les anticipations évoluent d'une période à l'autre. En conséquence, sa méthode ne permet pas d'analyser les effets à long terme d'une politique où la séquence d'équilibres qui en résulte :

"It is, indeed, not impossible to say something about further effects; for we can deduce what the stocks of goods will be at the end of the period if the decisions are carried out, and this gives us a basis for the analysis of a second period. But it is probable that the change in actual production during the first period will influence the expectations ruling at the end of that period; and there is not means of telling what that influence will be. The more we go into the future, the greater this source of error, so that there is a danger, when it is applied to long periods, of the whole method petering out." (1936: 241, souligné par nous)

Ce défaut de la méthode de Keynes est très certainement un problème que Hicks affronte luimême, au moment où il écrit, en préparant Valeur et capital. C'est le problème qu'il ne parviendra pas à résoudre. Il souligne d'ailleurs que ses « remarques à propos de la méthode générale des anticipations » ne «s'appliquent pas seulement au travail de M. Keynes mais aussi à tous les usages dont cette méthode a été l'objet dans le passé ou le sera dans le futur » (1936 : 240-1). L'intérêt de ce texte vient de ce que Hicks semble considérer le problème comme insoluble par nature :

"It is unrealistic to assume that an important change in data - say the introduction or extension of a public works policy - will leave expectations unchanged, even immediately. But this generally means only that there is a psychological unknown, affecting the magnitude of the impact effect. As more time is allowed, more and more scope is allowed for such variations, both in degree and kind. We must not expect the most elaborate economic analysis to enable us to see very far ahead » (1936: 241, souligné par nous).

L'état des anticipations constitue une «inconnue psychologique » que même «l'analyse économique la plus élaborée » ne serait pas en mesure de percer. Nous retrouvons l'idée selon laquelle la formation des anticipations échapperait au domaine de la théorie pure ${ }^{19}$.

Cette sorte de défaitisme confirme notre lecture des derniers chapitres de Valeur et capital. Toute l'analyse de l'équilibre temporaire et de son instabilité appelle une théorie de l'évolution inter-périodique des anticipations que Hicks lui-même juge impossible. La piste d'une représentation du cycle fondée sur les anticipations rencontre une impasse. Hicks le

\footnotetext{
${ }^{19}$ On peut se demander si nous ne nous trouvons pas ici confronté à l'élément marshallien de l'épistémologie hicksienne, une épistémologie qui fonctionne comme une échappatoire face aux exigences de l'approche walrassienne ou de «l'analyse logique pure du capitalisme ». Hicks n'a pas cherché à développer davantage son analyse des anticipations parce qu'il considérait qu'une théorie plus élaborée impliquait nécessairement une approche «mécanique ». Formaliser le lien entre les prix anticipés et les grandeurs de l'économie aurait conduit à un dangereux réductionnisme. Le chapitre 9 de Valeur et capital en offre un autre exemple. Comme le montre De Vroey (2006), l'exigence de réalisme conduit Hicks à rejeter l'hypothèse du tâtonnement walrassien et à supposer comme Marshall, et de façon incohérente, que des échanges ont lieu durant la formation des prix d'équilibre.
} 
reconnaît d'une certaine façon dans le chapitre 6 de Capital and Growth (1965) en répétant son jugement de 1936 à propos de l'approche de Keynes :

"But it is not dynamics. It is not the analysis of a process; no means has been provided by which we can pass from one Keynesian period to the next. (...). The Temporary Equilibrium model of Value and Capital, also, is 'quasi-static' - in just the same sense. The reason why I was contented with such a model was because I had my eyes fixed on Keynes » (1965: 65).

On comprend alors pourquoi Hicks a délaissé cette piste au profit d'approches moins dépendantes des anticipations en 1950 et en 1965.

\subsection{Un modèle acceptable peut-il faire une place à l'instabilité en un point du temps?}

L'examen des difficultés impliquées par le projet que Hicks formule en 1939 exige un retour sur la notion d'instabilité.

\subsubsection{L'absence d'outils mathématiques appropriés}

La théorie envisagée par Hicks bute évidemment sur un obstacle fondamental. Comme le montre l'introduction de l'appendice de Valeur et capital, pour Hicks, la méthode scientifique en économie passe par la démonstration mathématique. Or une telle démonstration des thèses concernant la stabilité de l'équilibre temporaire lui fait défaut.

L'économiste était nécessairement conscient de cette limite. En 1937, il publie en français une «Théorie mathématique de la valeur en régime de libre concurrence » qui présente une démonstration mathématique des résultats qui figureront deux ans plus tard dans les deux premières parties de Valeur et capital (théorie du consommateur et stabilité de l'équilibre général statique). Il conclut alors en affirmant que son analyse « est applicable à de nombreux problèmes dynamiques sans aucun changement dans l'attirail mathématique dont nous avons usé » (1937 : 54). Il annonce ensuite la parution prochaine de Value and Capital. L'extension de l'analyse mathématique des conditions de stabilité de l'équilibre général à l'équilibre temporaire était donc au cœur des préoccupations de Hicks à la fin des années 1930. La parution de l'article de Samuelson (1941) soulignant le caractère inadéquat de la méthode qu'il employait dans le cas statique a sans doute accru son embarras. En réalité bien sûr, Hicks ne démontre pas la stabilité de l'équilibre statique. Son approche est insuffisante et n'obtient la stabilité qu'en imposant des restrictions très forte sur les préférences des agents.

La conscience aiguë d'un défaut d'outillage dans la dernière partie de Valeur et capital est aussi attestée par une remarque adressée à Oskar Lange dans une recension de Price Flexibility and Employment (1944). Dans ce livre, Lange reprend et approfondit l'analyse en prose des conditions de stabilité de l'équilibre temporaire de Valeur et capital. L'exposé est conclu par un appendice mathématique dans lequel Lange prétend étayer ses thèses en s'appuyant sur la méthode d'analyse dynamique de Samuelson. Mais comme le souligne Hicks, le résultat n'est pas satisfaisant :

"A large part of this [mathematical] appendix is concerned with the relation between the stability conditions of the equilibrium analysis used in the text and the stability conditions of the corresponding process analysis worked out by Professor Samuelson. (It would have been interesting if some of the argument in the text could have been reworked on the Samuelson model) »(Hicks, 1945: 241, souligné par nous).

Hicks souligne discrètement le fait que l'appendice mathématique, inspiré par Samuelson, se réduit à l'analyse d'un système d'équilibre général dépourvu de monnaie, de titre et d'anticipations, très loin du modèle d'équilibre temporaire étudié dans le corps de l'ouvrage. 
Pour finir, l'ouvrage sur la théorie du cycle de 1950 ne marque pas seulement l'abandon du cadre walrassien au profit d'un cadre d'analyse keynésien. A la fin des années 1950, Hicks délaisse une approche de la dynamique qu'il n'est pas en mesure de développer sur le terrain mathématique. A l'inverse, l'outillage permettant l'analyse du modèle combinant le multiplicateur et l'accélérateur était accessible et son usage relativement balisé.

Mais des raisons encore plus fondamentales peuvent expliquer l'abandon du scénario d'instabilité dans les travaux ultérieurs de Hicks.

\subsubsection{Le caractère impensable de l'instabilité en un point du temps}

Nous avons montré plus haut (section 3.2.1.) comment l'importance qu'il accorde au scénario d'un équilibre temporaire instable place Hicks devant une contradiction. L'économiste base sa réflexion sur un cas qu'il juge à la fois irréaliste et incompatible avec la méthode de l'équilibre temporaire. La théorie des cycles qu'il esquisse dans la conclusion de son ouvrage offre une issue possible à cette contradiction. Mais l'ensemble du texte traduit un embarras persistant de l'auteur. Cet embarras est difficile à documenter. Nous nous contenterons de deux références au texte avant de revenir une dernière fois sur la signification de l'instabilité pour le théoricien.

Lorsqu'il en vient à présenter les hypothèses «which ought to be made in order to get a reasonably realistic model » $(1939: 271)$, Hicks n'écrit à aucun moment qu'il entend inscrire la possibilité de l'instabilité dans ce modèle. Comme je l'ai montré dans la partie 2, c'est pourtant bien ce qu'impliquent les hypothèses qu'il met en avant. Ensuite, dans le cadre de sa description raisonnée du cycle, en conclusion de l'ouvrage, Hicks explique comment les conditions d'un effondrement peuvent se trouver réunies dans la récession. Mais, bizarrement, il ne décrit ni ne discute le surgissement de l'instabilité et ses possibles conséquences sur l'économie. L'analyse s'arrête à l'évocation d' " une récession secondaire [...] beaucoup plus dangereuse que la première car il y a moins de résistance susceptible d'éviter l'effondrement » (1939: 298). Au fond, on ne sait toujours pas très bien en quoi consiste l'effondrement. Les prix tombent-ils à zéro ? Vont-ils fluctuer sans fin ? L'échange devient-il impossible?

Ce silence aux points cruciaux de l'exposé est étonnant. Nous l'interprétons de la façon suivante. Si Hicks ne dit rien sur l'effondrement lui-même, c'est simplement parce que la théorie s'arrête là où l'instabilité commence. La théorie walrassienne traite de l'équilibre, de ses caractéristiques et, éventuellement, de ses transformations au cours du temps. Elle n'a rien à dire au sujet d'un système qui ne parvient pas à atteindre l'équilibre ${ }^{20}$.

Au-delà, Hicks a certainement continué à se demander, comme en 1935, si le scénario d'instabilité n'était pas «too mad ». En 1939, il assimile l'instabilité à la mort du système capitaliste représenté par le modèle. Mais à quelle situation concrète ce scénario pouvait-il s'appliquer? N'était-il pas trop fort pour décrire le chaos économique connu dans les années 1930 par les Etats-Unis ou l'Allemagne ? Le concept de stabilité de l'équilibre temporaire peut sembler trop binaire, même pour décrire les effets d'une crise «catastrophique » sur une économie nationale. De surcroît, la perspective d'un effondrement du capitalisme au niveau mondial s'éloignait avec la fin de la seconde guerre mondiale et les premières années de l'âge d'or de la croissance.

En bref, pour Hicks comme pour la majorité des économistes, il y a quelque chose d'impensable dans l'instabilité de l'équilibre, quelque chose qui explique probablement pourquoi il a abandonné ce scénario dans ses travaux ultérieurs. La possibilité même de l'instabilité entre en conflit avec la conviction qu'une représentation réaliste et opérationnelle

\footnotetext{
${ }^{20}$ Un des rapporteurs a attiré notre attention sur l'attitude symptomatique de Walras lui-même dans les Eléments d'économie politique pure. Au détour d'une page, Walras mentionne la possibilité d'une crise mais passe à autre chose après trois lignes (Walras, $1988: 580$ ).
} 
de l'économie passe nécessairement par l'existence et la stabilité de l'équilibre général. Cette lecture est confirmée par un passage de la Contribution to the Theory of the Trade Cycle dans lequel Hicks explique pourquoi il présente le multiplicateur avant l'accélérateur :

"Since we do not want to waste our time in the contemplation of 'explosions' which in fact do not occur, it was as well to begin by familiarizing ourselves with some of the stabilizing forces »(1950: 37).

En 1950, l'effondrement économique, au cœur des derniers chapitres de Valeur et capital, est devenu un phénomène «qui en fait ne se produit pas » et, par conséquent, « une perte de temps ».

\section{Conclusion}

En rédigeant les derniers chapitres de Valeur et capital, Hicks semble avoir été fasciné par la possibilité théorique d'une instabilité de l'équilibre temporaire. Cette possibilité lui paraît offrir la clé de l'instabilité du système capitaliste telle qu'on peut l'observer dans la réalité, la clé, très certainement, de la dépression des années 1930. En ce sens, la réflexion hicksienne répond aux vœux émis par Krugman soixante-dix ans plus tard. Hicks prend pour point de départ de sa discussion du cycle la possibilité d'un « échec catastrophique dans une économie de marché ».

Le résultat est une proposition qui conserve un intérêt pour la théorie économique contemporaine. Cet intérêt vient du questionnement qu'elle implique. Une représentation générale de l'économie de marché devrait-elle tenir compte de la possibilité de son effondrement ou de son basculement dans le chaos? Si cette possibilité existe, nous devons comprendre pourquoi le système capitaliste est si résistant. D'une certaine façon, Hicks inverse ici le questionnement walrassien. Partant de l'instabilité inhérente au système capitaliste pur, il cherche à comprendre ce qui lui permet de fonctionner malgré tout. Hicks suggère qu'il existe des «stabilisateurs », c'est-à-dire des institutions comme la rigidité des salaires, l'éthique, la croyance en des prix normaux. Mais ces stabilisateurs sont fragiles. La foi en la stabilité du système, en particulier, peut être remise en cause par une crise particulièrement sévère. Si les institutions qui stabilisent l'économie sont liées à cette foi, c'est la structure même de l'économie qui peut être remise en cause par la crise.

Cependant, il y a loin de la coupe aux lèvres. Le passage du résultat d'instabilité à la théorie des cycles s'avère compliqué. Le modèle appelé par les derniers chapitres de Valeur et capital, suppose une endogénéisation de l'élasticité des anticipations. Hicks pose clairement le problème mais sa réponse ne dépasse pas le stade de l'ébauche. Non seulement il se heurte aux limites de sa capacité à formaliser son système d'équilibre temporaire, mais il semble qu'il ait refusé d'enfermer la psychologie des agents dans une représentation mécanique basée sur la théorie des choix. L'approche proposée par Hicks suppose un lien de dépendance particulier entre la rigidité des salaires et l'élasticité des anticipations. Comme nous l'avons montré, ce lien n'est pas correctement fondé. Enfin, l'instabilité de l'équilibre en un point du temps n'est pas le genre de résultat que l'économiste aime à manipuler. Hicks se demande si cette instabilité capture bien l'image de ce système qui plie mais ne rompt pas.

\section{Références}

Arena, R. (2001), "Hicks et la théorie du cycle d'affaire: une interprétation", Cahiers d'économie politique, $\mathrm{n}^{\circ} 39$, pp. 203-214. 
Bateman, B. W. (2003), "The end of Keynes and philosophy?" in S. Mizuhara, J. Runde, (éds.), Perspectives on the Philosophy of Keynes's Economics: Probability, Uncertainty, and Convention, London, New York, Routledge.

Benetti, C. (1998), "La structure logique de la Théorie générale de Keynes", Cahiers d'économie politique, $\mathrm{n}^{\circ}$ 30-31, pp. 11-52.

Benetti, C. (2001), "Monnaie, choix individuels et frictions", Cahiers d'économie politique, $\mathrm{n}^{\circ} 39$, pp. 89-106.

Béraud, A. (2008), "Allais et Hicks : de Walras aux modèles walrassiens", Communication à la $6^{\text {ième }}$ conférence de l'association internationale Walras, Kyoto, Japon.

Bliss, C. (1994), "Hicks on general equilibrium and stability", in: Hagemann, H. et O. F. Hamouda (éds), The Legacy of Hicks, His Contribution to Economic Analysis, London and New York: Routledge.

Boianovsky, M. (2004), "The IS-LM Model and the Liquidity Trap Concept: From Hicks to Krugman”, History of Political Economy, Annual Supplement to Vol. 36, pp. 92-126.

Coddington, A. (1983), Keynesian Economics. The Search for First Principles, London, Allen and Unwin.

De Vroey, M. (2004), Involuntary Unemployment, The elusive quest for a theory, London, Routledge.

De Vroey, M. (2006), "The temporary equilibrium method: Hicks against Hicks", European Journal for the History of Economic Thought, vol. 13 n ${ }^{\circ}$ 2, pp. 259-278.

Donzelli, F. (2009), "Hicks on Walrasian Equilibrium in the 1930s and Beyond", STOREP Sienna Workshop.

Dostaler, G. (2001), "De J. R. à John ou les métamorphoses de Hicks éléments de biographie intellectuelle", Cahiers d'économie politique, n³ 39, pp. 9-23.

Fisher, I. (1933), “The Debt Deflation Theory of Great Depressions”, Econometrica, n 1, pp. 337-357.

Grandmont, J. M. (1974), “On The Short Run Equilibrium in a Monetary Economy”, in J. Drèze (éd.), Allocation under Uncertainty, Equilibrium and Optimality, London, Macmillan.

Grandmont, J. M. (1983), Money and Value, Cambridge, Cambridge University Press.

Grandmont, J. M. (1991), "Temporary Equilibrium: Money, Expectations and Dynamics", in L. W. McKenzie, S. Zamagni, (eds.), Value and Capital Fifty Years Later, London, Macmillan.

Hicks, J. R. [1932] (1966), The Theory of Wages, New York, St Martin's Press.

Hicks, J. R. [1933](1982), "Equilibrium and the Cycle", Zeitschrift für Nationalökonomie, $\mathrm{n}^{\circ} 4$, traduction anglaise in Money, Interest and Wages, Collected Essays on Economic Theory, vol. 2, Oxford, Basil Blackwell, pp. 28-41.

Hicks, J. R. (1935a), "Wage and Interest: The Dynamic Problem", The Economic Journal, vol. $45 \mathrm{n}^{\circ} 179$, pp. 456-468.

Hicks, J. R. (1935b), “A Suggestion for Simplifying the Theory of Money”, Economica, New Series 2, pp. 1-19.

Hicks, J. R. (1936), “Keynes’ Theory of Employment”, The Economic Journal, vol. 46 n¹82, pp. 238-253.

Hicks, J. R. (1937), “Mr Keynes and the Classics: a Suggested Interpretation”, Econometrica, vol. $5 \mathrm{n}^{\circ} 2$, pp. 147-159.

Hicks, J. R. (1937), Théorie mathématique de la valeur en régime de libre concurrence, Paris, Hermann\&Cie.

Hicks, J. R. (1939) [1946], Value and Capital. An Inquiry Into Some Fundamental Principles of Economic Theory, $2^{\text {ième }}$ edition, Oxford, The Clarendon Press. 
Hicks, J. R. (1946), "Recent Contributions to General Equilibrium Economics", Economica, vol. $12 \mathrm{n}^{\circ} 48$, pp. 235-242.

Hicks, J. R. (1950), A Contribution to the Theory of the Trade Cycle, Oxford, The Clarendon Press.

Hoover, K. D. (1995), "Relative Wages, Rationality, and Involuntary Unemployment in Keynes's Labor Market”, History of Political Economy, vol. 27 n ${ }^{\circ}$, pp. 653-685.

Kaldor, N. (1951), "Mr. Hicks on the Trade Cycle", The Economic Journal, vol. 61 n $^{\circ} 244$, pp. 833-847.

Keynes, J-M. (1936)[1997], The General Theory of Employment, Interest and Money, San Diego, New York, London, Harcourt Brace\&Compagny.

Krugman, P. (2009), "How Did Economists Get it So Wrong?", The New York Times, 6 septembre.

Laidler, D. (1999), Fabricating the Keynesian Revolution, Studies of the Inter-war Literature on Money, the Cycle and Unemployment, Cambridge, Cambridge University Press.

Lange, O. (1944), Price Flexibility and Employment, San Antonio, Principia Press of Trinity University.

Walras, L. (1874)[1988], Eléments d'économie politique pure, Paris, Economica. 